\title{
Spoken Word Recognition: The Challenge of Variation
}

\author{
Paul A. Luce \\ University at Buffalo, The State University of New York, luce@buffalo.edu \\ Conor T. McLennan \\ Cleveland State University, c.mclennan@csuohio.edu
}

Follow this and additional works at: https://engagedscholarship.csuohio.edu/clpsych_facpub

Part of the Cognition and Perception Commons

How does access to this work benefit you? Let us know!

\section{Recommended Citation}

Luce, P.A. \& McLennan, C.T. (2005). Spoken word recognition: The challenge of variation. In Pisoni, D. B. \& Remez, R. E. (Eds.), Handbook of Speech Perception, pp. 591-609. Malden, MA: Blackwell.

This Contribution to Books is brought to you for free and open access by the Psychology Department at EngagedScholarship@CSU. It has been accepted for inclusion in Psychology Faculty Publications by an authorized administrator of EngagedScholarship@CSU. For more information, please contact library.es@csuohio.edu. 


\title{
24 Spoken Word \\ Recognition: The \\ Challenge of Variation
}

\author{
PAUL A. LUCE AND CONOR T. \\ MCLENNAN
}

\subsection{Introduction}

We are entering the fourth decade of research and theory devoted to understanding how listencers perceive spoken words. Although much has been learned, solutions (o) fundamental problems elude us (Jusczyk \& Luce, 2002), and even when consensus has been reached on answers to some of the basic questions, considerable dffort continues on further demonstrations of well-established phenomena that are accounted for by well-worn models or their variants (see, for example, Allopena, Magnuson, \& Tanenhaus, 1998; Gaskell \& Marslen-Wilson, 20(12). Certainly, theoretical refinement signals a mature science; stasis - be it in the form of apparently intractable problems or minimal progress on new research and theoretical foci - signals a paradigm in need of new challenges.

Consider the Trace model of spoken word recognition. Introduced in 1986, Trace (McClelland \& Elman, 1986) has been enormously influential, in part because it was the first connectionist model to attempt to account for spoken word processing. (As a measure of the model's influence, yearly average citation counts exceed most other widely cited theoretical papers in the field, including Marslen-Wilson \& Welsh, 1978; Marslen-Wilson \& Tyler, 1980; and Norris, 1994). Moreover, the model was instantiated in a computer simulation that has been widely available. One could either conduct simulations of the model to evaluate its predictions (Frauenfelder \& Peeters, 1990) or simply speculate about how it might account for data. Whatever the case, owing to its computational specificity, as well as its apparent ability to simulate a wide range of phenomena, Trace has dominated the theoretical landscape for years.

Despite its dominance, Trace has had its share of detractors. In fact, a number of competing models have been proposed. For example, Shortlist (Norris, 1994), PARSYN (Luce et al., 2000) and the Distributed Cohort Model (Gaskell \& MarslenWilson, 1997, 1999, 2002) attempt to overcome some of Trace's more glaring inadequacies. Nonetheless, all of these models for the most part attempt to account for the same basic empirical phenomena (although they may differ, sometimes subtly, in how they go about doing so; see Norris, McQueen, \& Cutler, 2000). 
In short, 18 years after its introduction, the Trace model and its descendants still monopolize the theoretical discussion. However, a number of compelling issues have arisen over the years that suggest that a new theoretical paradigm is in order. These issues - concerning both allophonic and indexical variation - are for the most part ignored by the dominant computational models, in part because their architectures are not easily amenable to the modifications required to account for these phenomena. In what follows, we focus on a selected subset of recent findings that suggest that we need to reconsider the way in which we think about representation and process in spoken word recognition. While we note our indebtedness to Trace and its ancestors for the insights they have provided us, we attempt to highlight new challenges to the theoretical zeitgeist.

To understand the emerging challenges to the dominant paradigm, we must, of course, understand the paradigm itself. Thus, we begin with a short tutorial on current computational models of recognition, after which we turn our focus to a selective discussion of some of the issues that have occupied our attention for the past few years, paying particular attention to those empirical issues that have direct bearing on the current batch of computational models. Having set the stage, we then consider some recent, still evolving theoretical and empirical issues that we suggest may provide new challenges, and hence new insights, into the nature of spoken word perception.

\subsection{Recent Models of Spoken Word Recognition}

\subsubsection{Trace}

The Trace model (McClelland \& Elman, 1986) is an interactive-activation, localist connectionist model of spoken word recognition that consists of three levels of primitive processing units - or nodes - that correspond to features, phonemes, and words. (In localist models of word recognition, individual processing units correspond to entities such as allophones, phonemes, or words.) Trace's processing units have excitatory connections between levels and inhibitory connections among levels, with the connections serving to raise and lower activation levels of the units depending on the stimulus input and the activity of the overall system. By passing activation between levels, the model serves to confirm and accentuate evidence in the input corresponding to a given feature, phoneme, and word. Moreover, lateral inhibition among units within a level enables winning units to suppress the activity of their competitors. ${ }^{1}$

Although Trace has had considerable influence, the model incorporates a decidedly questionable architecture. Its system of nodes and connections are duplicated over successive time slices of the input, a rather inelegant (and probably psychologically implausible) means of dealing with the temporal dynamics of spoken word recognition. 


\subsubsection{Shortlist}

Norris' (1994) Shortlist model, a descendant of Trace, is also a localist connectionist model of spoken word recognition. In the first stage of the model, a "shortlist" of word candidates is activated that consists of lexical items that match the bottomup speech input. In the second stage of processing, the shortlist of lexical items enters into a network of word units, much like the lexical level of Trace. Lexical units at this second level of processing compete with one another via lateral inhibitory links for recognition.

Shortlist simulates the temporal dynamics of spoken word recognition without resorting to the unrealistic architecture of Trace, in which single words are represented by a plethora of identical nodes across time. In addition, Shortlist attempts to provide an explicit account of segmentation of words from fluent speech via mechanisms of lexical competition. Finally, Shortlist is the current example of an autonomous model of recognition. Unlike Trace, Shortlist does not allow for top-down lexical influences on its phoneme units; flow of information between phoneme and word is unidirectional and bottom-up. Thus, the Shortlist model embodies the notion, which has received some empirical support (Burton, Baum, \& Blumstein, 1989; Cutler, Norris, \& Williams, 1987; McQueen, 1991), that the processing of phonemes in the input is autonomous of top-down, lexical influences (sec Norris et al., 2000, and the accompanying responses).

\subsubsection{PARSYN}

PARSYN (Luce et al., 2000) is a localist connectionist model with three levels of interconnected units: (1) an input allophone level, (2) a pattern allophone level, and (3) a word level. Connections between units within a level are mutually inhibitory. However, links among allophone units at the pattern level are also facilitative across temporal positions. Connections between levels are facilitative, also with one exception: The word level sends inhibitory information back to the pattern level, quelling activation in the system once a single word has gained a marked advantage over its competitors. The first, or input, layer consists of position-specific allophonic units arranged into banks of receptors corresponding to the temporal sequence of the input. The second, or pattern, layer of units exactly duplicates the input layer, with units at the pattern level receiving direct facilitative input from the allophone input units. However, the input and pattern layers differ in the interconnections between the units. Whereas banks of units at the input level do not directly interact over time, units at the pattern level receive facilitative input from other pattern layer units in preceding and/or following temporal positions. The weights on these within-level connections correspond to forward and backward position-specific transitional probabilities. In addition, resting levels of the pattern-layer nodes correspond to the position-specific probability of occurrence. The transitional probabilities and activation levels of allophone units are designed to represent the (first order) probabilistic phonotactic constraints of the words in English. The third layer consists of word units. Word level units receive facilitative input from their constituent position-specific 
allophones at the pattern level. Each word level unit is capable of inhibiting all the other word units.

PARSYN is aimed at simultaneously accounting for effects of lexical competition and probabilistic phonotactics (see below; also see Auer \& Luce, this volume). Moreover, unlike Trace and Shortlist, PARSYN proposes an intermediate allophonic - as opposed to phonemic - level of representation.

\subsubsection{Distributed cohort model}

In Gaskell and Marslen-Wilson's (1997, 1999, 2002) distributed cohort model (DCM), activation corresponding to a word is distributed over a set of simple processing units (i.e., the DCM is not a localist model). In particular, featural input based on speech input is projected onto simple semantic and phonological units. Because the DCM is distributed, there are no intermediate or sublexical units of representations. Moreover, lexical competition is expressed as a blending of multiply consistent lexical items based on bottom-up input, in contrast to the mechanism of lateral inhibition employed by the localist models.

\subsubsection{Some comparisons}

Trace, Shortlist, PARSYN, and DCM all assume that multiple form-based representations of words compete for recognition. The localist models each propose that word units are connected via lateral inhibitory links, enabling a unit to suppress or inhibit the activation of its competitors (see McQueen, Norris, \& Cutler, 1994). The degree to which a unit inhibits its competitors is proportional to the activation level of the unit itself, which is determined in large part by its similarity to the input. The DCM, on the other hand, proposes a blending model of lexical competition, in which increases in the number of phonologically similar words consistent with the input result in more diffusely activated distributed representations. Nonetheless, in all models, competitor activation is assumed to be a function of the degree of similarity of the competing words to the input.

Trace, Shortlist, and PARSYN posit sublexical levels of representation. In contrast, DCM explicitly eschews intermediate units (although, as is often the case in distributed models, these units may be emergent). However, each model to varying degrees suffers from a significant weakness in terms of how they map input onto their form-based representations, be they lexical or sublexical. In particular, the models rely on coding the acoustic-phonetic signal into either abstract phonetic features (in Trace or DCM) or phonemes (in Trace and Shortlist) that vary neither as a function of time, rate, phonological context, or talker. That is, the models ignore much of the contextual and temporal detail encoded in the signal. Although Trace allows for overlapping features in an attempt to capture effects of coarticulation, the features themselves remain unchanged by the context in which they occur. Whereas Shortlist holds out promise for more realistic input based on the output of a simple recurrent network, the model as implemented makes no use of context-dependent, sub-phonemic information in lexical processing. Although PARSYN's use of allophonic representations attempts 
to capture some context-dependency at the sublexical level, it too fails to make full use of the rich source of information embodied in the speech signal itself. Moreover, PARSYN's allophonic representational scheme may make it incapable of representing more abstract (perhaps phonemic) units (see below).

\subsection{Core Issues: Activation and Competition}

Much to its credit, the research on the core issues in spoken word recognition has gone hand-in-hand with theory and model development. Indeed, one can see an intimate link between the theories we just discussed and the core empirical issues of activation and competition. Virtually all current models of spoken word recognition share the assumption that the perception of spoken words involves two fundamental processes: activation and competition (see Gaskell \& Marslen-Wilson, 2002; Luce \& Pisoni, 1998; Marslen-Wilson, 1989; McClelland \& Elman, 1986; Norris, 1994). Although there is some consensus that input activates a set of candidates in memory that are subsequently discriminated among, details of the activation and competition processes are still in dispute.

\subsubsection{Activation}

Current computational models of spoken word recognition all ascribe, to varying degrees, to the notion of radical activation. These models (e.g., Trace, Shortlist, PARSYN, and - at least in principle - DCM) propose that form-based representations consistent with stimulus input may be activated at any point in the speech stream. The notion of radical activation differs from various earlier proposals that initial activation of lexical items is restricted to word onsets (as in the earliest version of cohort theory: Marslen-Wilson \& Welsh, 1978) or stressed syllables (Cutler \& Norris, 1988). According to radical activation models, spoken input corresponding to $\operatorname{dog}$ may activate bog based on the overlapping vowel and final consonant, despite the fact that the two words differ initially. Of course, most radical activation models afford priority to $d o g$ in recognition process, primarily because of the relative temporal positions of the mismatch and overlap. Furthermore, in the localist models, lateral inhibition at the lexical (and sometimes sublexical) levels typically grants considerable advantage to representations overlapping at the beginnings of words. Nevertheless, radical activation models propose that any consistency between input and representation may result in some degree of activation.

Evidence for radical activation abounds. For example, Connine, Blasko, and Titone (1993) found facilitative priming effects between rhyming nonword primes and real word targets, suggesting that activation of competitors is not limited to overlapping word-initial information. The conclusion that competitor activation depends on initial overlap is also contradicted by a series of intra-modal formbased priming studies (Goldinger, Luce, \& Pisoni, 1989; Goldinger et al., 1992; Luce et al., 2000). In one of these studies, Luce et al. presented participants with primes and targets that were phonetically similar but shared no position-specific segments (e.g., shun-gong). The participants' task was to shadow the target word. 
Luce et al. found that shadowing times were significantly slower for targets following phonetically related primes than to ones following unrelated primes. This result is consistent with the radical activation account, given that none of the prime-target pairs shared word-initial segments. Moreover, the finding that phonetically related primes actually slowed, rather than facilitated, response times provides direct support for the activation-competition framework, which states that similar form-based representations compete for recognition.

Allopena et al. (1998) provide additional support for radical activation models. Using a head-mounted eye tracker with which participants' eye movements could be monitored as they followed spoken instructions to manipulate objects on a computer screen, Allopena et al. found that rhyming competitors are activated early in the recognition process. When asked to use a mouse to click on a picture of a beaker, participants' fixation probabilities indicated that they also considered a picture of a speaker to be a likely candidate. These findings indicate that shared word-initial information is not necessary to activate competitors.

The preponderance of the evidence has led to a general consensus that spoken word recognition is best modeled as a process of activation of multiple word forms that are consistent with the input. Moreover, this activation process appears to be radical, in that consistencies between input and representation at any point in time may - at least in principle - result in activation of lexical items in memory.

\subsubsection{Competition}

In activation-competition models, the hallmark of the lexical recognition process is competition among multiple representations of words activated in memory. As a result, the role of competition has been a primary focus of research and theory on spoken word recognition in the last few years (e.g., Cluff \& Luce, 1990; Gaskell \& Marslen-Wilson, 2002; Goldinger et al., 1989; Marslen-Wilson, 1989; McQueen et al., 1994; Norris, McQueen, \& Cutler, 1995; Vitevitch \& Luce, 1998, 1999).

Evidence for competition among form-based lexical representations activated in memory has come from a variety of experimental paradigms. For example, Luce and colleagues (Cluff \& Luce, 1990; Luce \& Pisoni, 1998) have shown that similarity neighborhood density and frequency, both indices of lexical competition, have demonstrable effects on processing time and accuracy in speeded single-word shadowing, auditory lexical decision, and perceptual identification. A similarity neighborhood is defined as a collection of words that are similar to a given target word. Neighborhoods may vary on both the density and frequency of the words that comprise them. Luce and colleagues have shown that words residing in densely populated similarity neighborhoods, in which lexical competition is predicted to be strong, are processed less quickly and less accurately than words residing in sparsely populated neighborhoods. Moreover, in similarity neighborhoods composed of high-frequency words, competition is more severe than in neighborhoods of low frequency words, resulting in slower and less accurate processing.

Although there is now considerable evidence for competitive effects in spoken word recognition, some debate remains over the precise mechanisms underlying lexical competition. As noted above, in models of recognition such as Trace, 
Shortlist, and PARSYN, lateral inhibition among lexical representations is a fundamental feature of the competitive process. The DCM, on the other hand, eschews the notion of lateral inhibition in favor of a competitive process that results from the blending of multiple distributed representations (Gaskell \& Marslen-Wilson, 1997, 1999, 2002). At present, there is no definitive evidence to help distinguish between these accounts of lexical competition (see, however, Gaskell \& Marslen-Wilson, 2002).

\subsubsection{Activation-competition models}

When considered within a larger context, the differences among the current batch of activation-competition models appear to be rather minor. Indeed, the less jaundiced eye might see remarkable unanimity among the models. For example, all agree that spoken word recognition is characterized by multiple activation of and competition among form-based lexical items. Although details may vary, the basic facts appear to have been established. Admittedly, there are other phenomena addressed by the models, for example, segmentation, lexical embeddedness, the nature of lexical feedback, and the role of context, to name a few. However, given the fundamental similarity of the current models, it is doubtful that any of these issues will prove to be determinative in deciding which model should prevail, especially given that fixes and additions are always in the offing (see, for example, the Merge model, Norris et al., 2000).

Wo should view the current state of theoretical affairs as an indication that we are converying on some basic truths and that the science aimed at understanding spoken word perception is maturing. However, new insights tend not to spring from consensus but from challenges. We now turn to two exciting areas of research that have emerged over the past few years that pose just such challenges to the current theoretical status quo: processing and representation of indexical and allophonic variation. We argue that these recent research foci, which are largely ignored by the current models, demand our attention. Indeed, each of these areas of research may lead us to a new conceptualization of spoken word process and representation.

\subsection{Challenges: Variation in Spoken Word Recognition}

\subsubsection{Indexical variation}

Each of the theories of spoken word recognition we have discussed assumes that lexical items are represented in memory by abstract phonological codes that only preserve information relevant for lexical discrimination. Indexical variation arising from differences in speaking rate, differences among talkers, differences in affective states, and so on - is treated as irrelevant information that is discarded early in the encoding process (i.e., the input is normalized). Trace, Shortlist, PARSYN, and DCM propose that input is mapped onto abstract features, 
allophones, phonemes, or some combination of the three, which are then used to contact form-based lexical representations. However, spoken words may differ on many physical dimensions not captured by these abstract units, and these dimensions may have demonstrable consequences for lexical representation and process.

Recent research has suggested that putatively irrelevant surface details of words - such as information specific to a given talker - are preserved in some form in memory (see Goldinger, 1996, 1998; Pisoni, 1997, for reviews). The findings regarding specificity effects have led to the proposal that lexical items are represented in memory by representations that preserve, rather than discard, much of the physical detail of the stimulus (Goldinger, 1996, 1998). Specifically, this research has examined the effects of indexical variation on spoken word processing and representation.

\subsubsection{Indexical variation and processing}

Variation in the surface details of spoken stimuli has pronounced implications for spoken word processing. According to Pisoni (1992a), the earliest research to investigate processing costs due to talker variability (one form of indexical variation) was carried out by Peters (1955) and Creelman (1957). Peters compared the intelligibility of single-talker and multiple-talker messages in noise. He found that single-talker messages were reliably more intelligible than multiple-talker messages. Creelman compared the intelligibility of words spoken by either a single talker or by multiple talkers and found an inverse relationship between identification performance and the number of talkers: As the number of talkers increased, identification performance decreased.

In the late 1980s, Pisoni and his colleagues revisited the effects of talker variability on spoken word perception. Mullennix, Pisoni, and Martin (1989) examined participants' identification performance for English words spoken by either a single talker or by multiple talkers. Replicating the earlier work by Peters and Creelman, they found that participants' identification performance was more accurate in the single-talker than in the multiple-talker condition. Likewise, Mullennix et al. also found that participants were not only less accurate but also slower to repeat words in lists containing multiple talkers compared to lists produced by a single talker.

A number of other studies have demonstrated performance costs (measured in terms of decreased accuracy, increased reaction times, or both) associated with processing words spoken by multiple talkers, relative to a single-talker (see Goldinger, Pisoni, \& Logan, 1991; Martin, Mullennix, Pisoni, \& Summers, 1989; Palmeri, Goldinger, \& Pisoni, 1993; also see Pisoni, 1990, 1992b). For example, similar findings have been obtained in preschool children (Ryalls \& Pisoni, 1997) and hearing-impaired adults (Kirk, Pisoni, \& Miyamoto, 1997). Research has also demonstrated that changes in talkers affect the perception not only of words but of speech segments themselves. For example, identification of vowels (Verbrugge et al., 1976) and consonants (Fourcin, 1968) is more accurate when they are produced by a single talker than when they are produced by multiple talkers.

Clearly, perception of both segments and words is directly affected by indexical variation. However, this important observation has yet to be acknowledged in 
current models of recognition. In fairness, these models have restricted their domain of focus to issues such as activation, competition, and segmentation. Nonetheless, the pervasive effects of indexical variability on spoken word perception suggest that an adequate model of recognition must have some mechanism for accounting for the sensitivity of the perceptual system to lexically irrelevant variation. Of course, the answer may simply be that prelexical normalization processes which are not within the explanatory domain of current computational models of word recognition - reduce resources available for encoding, rehearsal, or both, thus producing processing deficits in the face of indexical variation. That is, a front-end model of normalization in speech perception interfaced to Trace, Shortlist, PARSYN, or DCM may be sufficient for explaining specificity effects on processing. ${ }^{2}$

On the other hand, processing effects on indexical variation may require that the representational schemes embodied in current models be re-examined (see, for example, Remez, Fellowes, \& Rubin, 1997). In particular, do specificity effects indicate that lexical and sublexical representations themselves are highly specific, or - more likely - that these representations adapt or retune themselves to each encounter with the speech stimulus? If those representations responsible for spoken word processing are plausibly implicated in effects of indexical variation, our current models are inadequate. In short, the implications of indexical variation effects may be deep and may force us to rethink computational models consisting of abstract sublexical and lexical nodes.

\subsubsection{Indexical variation and representation}

A more serious challenge to current models comes from research on the representation of indexical variation, in particular from research using the long-term repetition priming paradigm (Church \& Schacter, 1994; Goldinger, 1996; Luce \& Lyons, 1998; Schacter \& Church, 1992; Sheffert, 1998). This paradigm has enabled investigators to examine the degree of specificity and abstractness of form-based representations, which has in turn provided new insights into the architecture of the word recognition system. Investigators have used the phenomenon of long-term, form-based repetition priming to determine the degree to which lexical representations encode the variability inherent in spoken words.

The logic of the repetition paradigm is simple: Processing of a spoken word (as measured by accuracy, processing time, or both) is facilitated when the word is repeated exactly. However, if the first and second presentations (prime and target, respectively) mismatch on some dimension, the priming effect is often attenuated. We can infer from a reduction in priming that the prime and target activate somewhat different specific form-based lexical representations. If, on the other hand, the priming effect is unaffected by any differences between the prime and target, we can conclude that the prime and target activate the same underlying representations.

Church and Schacter (1994) and Schacter and Church (1992) observed effects of talker variation in implicit tasks such as fragment completion and identification of low-pass filtered stimuli. Participants were more likely to complete a fragment of a word if it was repeated in the same voice. Participants were also more accurate at identifying low-pass filtered words that were repetitions of previously 
presented items if the repetition preserved surface characteristics of the stimulus. Goldinger (1996) presented words in recognition and perceptual identification tasks with varying delays between prime and target and found significant effects of voice in both recognition and identification. In another experiment, he demonstrated that effects of voice varied with level of processing, such that strongest effects of stimulus specificity were observed in the shallower processing conditions, especially for recognition memory.

Luce and Lyons (1998) examined the effects of changing voice on stimulus repetition in both auditory lexical decision and recognition memory tasks (Biederman \& Cooper, 1992; Cooper et al., 1992). They first presented participants with a list of stimuli spoken by two talkers in a lexical decision task. They followed this first block of lexical decision trials with either (1) another block of lexical decision trials (implicit task) or (2) a block of old/new recognition trials (explicit task). The stimuli in the second block of the experiment were either repeated in the same voice, a new voice, or were new items that had not appeared in the first block.

Luce and Lyons demonstrated that repetition priming for spoken words might not always be sensitive to changes in the surface characteristics of the stimuli. When participants were required to make lexical decisions to spoken words in the second block of trials, response times to repetitions in the same voice were not statistically different from response times to repetitions in the different voice, although overall effects of repetition priming were robust. However, in the explicit old/new recognition memory experiment, they obtained significant effects of voice: Participants responded old more quickly to words repeated in the same voice than to words repeated in the different voice. The results of Luce and Lyons' explicit old/new recognition task are consistent with the previous demonstrations that voice matters in recognition memory. However, the failure to observe specificity effects in the implicit priming task does not replicate previous work.

Luce, McLennan, and Charles-Luce (2003) have proposed that the failure of Luce and Lyons to observe specificity effects in lexical decision lay in the rapidity of the response, a proposal they dubbed the time course hypothesis. Compared to off-line identification, responses in the lexical decision task may be so rapid as to precede potentially slower acting effects of stimulus specificity in processing (Hintzman \& Caulton, 1997; Hintzman \& Curran, 1997).

Evidence for the time course hypothesis comes from a number of sources. For example, Goldinger (1996) reports one of the few spoken word recognition studies that has examined response latencies in which voice was manipulated. Response latencies to classify stimuli in his fastest condition were almost $100 \mathrm{~ms}$ longer than the latencies in Luce and Lyons' priming task. Thus, it may be that if participants are capable of making an identification decision quickly enough, effects of stimulus specificity will be small. Conversely, when responses are slower, as in Luce and Lyons' old / new recognition experiment or in Church and Schacter's and Goldinger's studies, effects of voice emerge. (See also Mullennix et al., 1989, and Goldinger et al., 1991).

Further support for this hypothesis comes from a study by McLennan, Luce, and Charles-Luce (2003). In contrast to the stimuli used by Luce and Lyons, which were short consonant-vowel-consonant words with a fairly high average frequency, McLennan et al. examined specificity effects for longer, lower-frequency 
bisyllabic spoken words (again presented in the clear for a speeded response). All things considered, Luce and Lyons' short, higher frequency stimuli should be recognized faster than McLennan et al.'s longer, lower-frequency stimuli. If the time course hypothesis is viable, specificity effects should emerge for those stimuli requiring longer processing times. Indeed, the average processing times to target stimuli in McLennan et al.'s study was $65 \mathrm{~ms}$ longer than the average lexical decision times reported by Luce and Lyons and, as predicted, large effects of specificity were observed.

Taken together, the Luce and Lyons and McLennan et al. results suggest that specificity effects may take time to develop. If we are able to tap into the perceptual process early, by examining processing of short, high-frequency words in a speeded task, no effects of indexical variability are observed. However, specificity effects on long-term priming are clearly in evidence when perception is slowed, even in a speeded perceptual task.

\subsubsection{Summary}

Although somewhat varied, the overall results of studies examining the effects of voice on identification and memory are consistent with exemplar-based (e.g., Hintzman, 1986) or distributed representations that encode lexically irrelevant information. According to these models, variation is encoded directly as changes in representations - taking the form of new exemplars or subtle changes in connection weights in distributed representations. An advantage of these types of models is that they have the potential for solving the long-standing problem of perceptual normalization in speech perception by dispelling the notion that the ultimate goal of the perceptual process is to map acoustic-phonetic information onto abstract form-based representations of words in memory. In exemplar-based and certain distributed models, the representational currency of the perceptual encoding process is more-or-less true to the details of the stimulus itself. If correct, current computational models fail in their representational assumptions.

The time course of specificity effects also poses a significant challenge to current computational models. If current models cannot account for effects of indexical information on both processing and representation, they are certainly inadequate as models of the time course of specificity effects. The results we have just discussed suggest a system in which rapid recognition may proceed based on abstract codes untainted by surface variation. However, slight delays in processing - as encountered when attempting to identify bisyllabic words - may afford the opportunity for indexical information to exert its influence.

The consequences of encoding lexically irrelevant information directly into sublexical and lexical representations may lead us toward new models with substantially different architectures. For example, the work on long-term repetition priming (and, to a lesser extent, that on processing of indexical information) demonstrates that the perceptual system is highly adaptive, constantly tuning itself to changing environment stimulation: Under the appropriate circumstances, representations may reflect the details of words last encountered. If adaptation is indeed fundamental to word perception, the current cadre of computational models may fail not only to account for the adaptive nature of the system, these models also may be substantially in error in their proposed representations and 
architecture: Systems of interconnected nodes corresponding to abstract sublexical and lexical representations may be poor approximations to reality.

\subsubsection{Allophonic variation}

Our thesis in this chapter is that accounting for variability in spoken word recognition poses a specific challenge to our current models. We believe that this case is amply supported by the research on representation and process of indexical variation in spoken word recognition. However, recent work on allophonic variation suggests further inadequacies in the current models. Whereas indexical variability refers to variations in a spoken word that arise from differences among talkers, speaking rates, affective states, and so on (Abercrombie, 1967; Pisoni, 1997), allophonic variation refers to articulatory and acoustic differences among speech sounds belonging to the same phonemic category (Ladefoged, 2000). ${ }^{3}$ For example, the stop consonant $/ \mathrm{p} /$ is articulated somewhat differently before a vowel (as in pot), after a vowel (as in top), and in a consonant cluster (as in spot). Each of these different versions are referred to as allophones of the phoneme $/ \mathrm{p} /$. Recent research on allophonic variation has led to further insights into the potential inadequacies of current modeling approaches.

Traditionally, spoken word perception has been characterized as being comprised of a series of linguistic stages of analysis, with form-based representations becoming successively more abstract at each stage of processing. This view of mediated lexical access finds its expression in Trace, Shortlist, and PARSYN. Recently, these mediated access models have been challenged by direct access models, which state that after the initial recoding of sensory data, information is mapped directly onto form-based lexical representations. For example, the DCM proposes that lexical representations are accessed directly from phonetic features. In short, although both mediated and direct access theories assume that sensory information is initially recoded in some manner, they differ as to whether additional levels of representation intervene between sensory recoding and lexical representation.

Evidence in support of direct access models comes from a series of experiments reported by Marslen-Wilson and Warren (1994; see also Whalen, 1984, 1991, and Streeter \& Nigro, 1979). Marslen-Wilson and Warren examined processing for a set of cross-spliced words and nonwords containing subcategorical mismatches. They observed processing costs only when mismatching coarticulatory information involved words. However, nonwords cross-spliced with other nonwords failed to exhibit processing costs associated with subcategorical mismatch. MarslenWilson and Warren concluded that the failure to find effects of subcategorical mismatch for nonwords is due to the absence of intermediate representations that could detect the subcategorical mismatch.

Recently, McQueen, Norris, and Cutler (1999) challenged Marslen-Wilson and Warren's finding. They found that the crucial distinction between words crossspliced with other words and nonwords cross-spliced with other nonwords could be made to come and go as a function of task demands. Moreover, they found that models with a phonemic level of representation could simulate the data pattern obtained by Marslen-Wilson and Warren, thus calling into question the 
claim that mediated models should always show effects of conflicting information at a sublexical level. Nonetheless, a lack of positive evidence for sublexical representations persists. As a result, the debate between mediated and direct access theories remains unresolved.

We recently examined the status of intermediate representations in more detail by exploring the perceptual consequences of allophonic variation (McLennan et al., 2003). More specifically, we examined flapping in American English. A flap $(/ f /)$ is a neutralized version and allophone of intervocalic $/ t /$ and $/ d /$. In casually produced American English, when a / $t /$ or a $/ d /$ is produced between two vowels, as in greater or Adam, it is often realized as a flap, a segment that is neither exactly a / $/$ / nor exactly a /d/ (see Patterson \& Connine, 2001). We attempted to determine if flaps map onto their underlying, abstract phonemic counterparts, /t/ and /d/. Mediated access theories predict that allophonic variation occurring on the surface should map onto more abstract, underlying phonological representations (see e.g., Pisoni \& Luce, 1987). However, according to direct access theories, allophonic variation occurring on the surface should map directly onto lexical representations. Therefore, examining the perceptual consequences of allophonic variation may help to distinguish between these competing theories.

We used the long-term repetition priming paradigm to determine if flapped segments are mapped onto underlying intermediate form-based representations of $/ \mathrm{t} / \mathrm{s}, / \mathrm{d} / \mathrm{s}$, or both, or if flaps are represented veridically as they appear in casual speech as $/ \mathrm{r} /$. In particular, we attempted to determine if the surface allophonic representation, $/ \mathrm{r} /$, is recoded into the underlying phonological representations, $/ \mathrm{t} /$ or $/ \mathrm{d} /$, as predicted by mediated access theories of spoken word recognition.

In this set of experiments, two blocks of stimuli containing carefully and casually articulated versions of words were presented. Casually articulated (hypoarticulated) words are produced in a relaxed manner, whereas carefully articulated words are more clearly articulated. Intervocalic /t/s and /d/s are flapped in casually articulated words but not in carefully articulated words. We hypothesized that priming of casually articulated stimuli by carefully articulated stimuli (or vice versa) would indicate the presence of a mediating underlying representation in memory. The presence of specificity effects - in which flaps fail to prime carefully articulated segments, and vice versa - indicates the absence of intermediate representations, consistent with direct access theories. Conversely, lack of specificity effects indicates the presence of intermediate representations, consistent with mediated access theories.

To review, Trace, Shortlist, and PARSYN all assume that access to the lexicon is mediated by intervening representations. Direct access theories, such as the DCM, assume that following initial sensory registration, access to the lexicon is direct. Thus, these classes of theories make opposite predictions regarding the perceptual consequences of allophonic variation.

The results of a series of repetition priming experiments were not entirely consistent with either mediated or direct access models, suggesting that the dichotomy represented by Trace, Shortlist, and PARSYN on the one hand and the DCM on the other may fail to capture the underlying nature of the representational and processing system devoted to spoken word perception. In our initial 
experiments, we found that flapped words primed carefully articulated words as much as carefully articulated words primed themselves, a result consistent with mediated access models. However, much like the effects of indexical variation discussed above, degree of priming from flapped to carefully articulated words varied as a function of the time course of processing. In general, when participants responded relatively slowly, we found evidence for the activation of underlying representations. However, when we manipulated the experimental conditions in such a way as to encourage more rapid responding, we observed no evidence for the activation of mediating representations. ${ }^{4}$

Overall, we demonstrated that underlying representations appear to dominate processing when spoken input is phonologically ambiguous (i.e., when flaps are present) and when enough time is allowed for the underlying representations to have an effect on recognition. Alternatively, surface representations appear to dominate processing when spoken input is unambiguous and when there is little time for the underlying representations to have an effect on recognition.

No current computational model of spoken word recognition is capable of capturing this pattern of results. For example, Trace and Shortlist both lack an allophonic layer of representation, a minimal requirement dictated by the finding that under appropriate circumstances flaps activate their phonemic counterparts. Only PARSYN incorporates an explicit allophonic level. However, PARSYN lacks phonemic representations, which may prove problematic in accounting for the activation of underlying forms (although PARSYN's lexical representations are phonemically coded). In addition, although certain of the mediated access models may account for the finding that underlying representations are activated, they appear incapable of providing an account of the time course of processing, namely that when responses are rapid, effects of underlying representations are absent. Finally, although the DCM can account for those situations in which underlying representations are not activated, the model will probably be hard pressed to simulate activation of underlying representations when processing is slowed. Once again, the current cadre of models fails to meet the challenge posed by variation.

Noting the apparent inability of the current computational models to account adequately for the findings on the representation and processing of allophonic variation, we proposed an account of these findings based on Grossberg's ARTPHONE model (Grossberg, Boardman, \& Cohen, 1997; see also Vitevitch \& Luce, 1999). According to this model, acoustic-phonetic input comprised of relatively veridical surface representations resonate with chunks corresponding to more abstract phonological representations, as well as to chunks corresponding to less abstract, allophonic representations. In the absence of ambiguity in the input, the resonances between surface forms and chunks corresponding to underlying representations preserve detail (see Grossberg \& Myers, 2000). However, underlying representations (or chunks) activated by ambiguous input (i.e., flaps) may result in a restoration of surface representations not actually in the input (i.e., underlying / $t /$ and /d/). Furthermore, the restoration of surface representations by the underlying chunks requires time. Thus, tasks that tap into the recognition process prior to restoration of the surface representation should fail to show effects of underlying abstract representations, presumably because the underlying representations have not had sufficient time to establish resonance with a 
restored surface form. In short, the adaptive resonance framework is able to account for both the coexistence of specific and abstract representations and the relative speed with which they influence processing.

\subsection{Conclusion}

We began our discussion with the observation that the scientific endeavor aimed at understanding how listeners perceive spoken words appears to have reached a plateau, concerning itself with refinements and extensions of well-worn models that account for many of the major phenomena in the field. We have argued that challenges to the existing theoretical paradigm already exist, in the form of research on indexical and allophonic variation, and that these challenges may lead to the next generation of models of spoken language perception.

The challenges posed by variation are fundamental: We need to rethink the representational schemes of our models. The emerging evidence suggests the coexistence of representations that encode both the specific and the abstract. Moreover, we must conceive of systems in which processing of the specific and abstract follows a predictable time course, a time course that reflects the underlying architecture of the processing system itself. Finally, our next generation of models must appreciate the adaptive nature of perception. Even adult brains appear to tune, finely and frequently, to environmental stimulation. Adequate models of recognition must incorporate representational systems that can account for the adaptive nature of perception, and such an account will certainly have deep implications for the nature and architecture of the representational system itself.

Our belief is that the adaptive resonance framework outlined above is a good starting point: It does not propose a rigid hierarchy of abstract sublexical and lexical nodes, it explicitly incorporates a learning component that leads to constant tuning of representations to input, and it has the capability (although largely unexplored) of addressing the challenge of variation.

\section{ACKNOWLEDGMENTS}

This work was supported (in part) by research grant number R01 DC 0265801 from the National Institute on Deafness and Other Communication Disorders, National Institutes of Health. We thank Jan Charles-Luce for her assistance and helpful advice.

\section{NOTES}

1 This version of Trace as well as the other computational models discussed here use various coding schemes to abstractly represent the phonetic input to the models.
2 Specifying the precise nature of this initial prelexical process - which must somehow discard irrelevant variation by mapping specific information on to more abstract, canonical features or 
segments - may be itself prove to be an enterprise equal in scope to the lexical processing models themselves. See Mullennix et al. (1989) for discussion.

3 Allophonic variation may arise from the predictable interaction of the articulators, and thus may be systematic across languages, or may be dictated or allowed by the phonology, thus being dialect- or languagespecific.

4 It may appear that we are making contradictory proposals about the time course of specificity, in particular, that processing of indexical specificity lags behind activation of more abstract representations, whereas processing of allophonic specificity precedes activation of underlying representations. We point out that in our framework, allophonic information is only specific relative to underlying abstract phonemic representations; allophonic variation itself constitutes fairly abstract information, especially compared to indexical variability (see, however, Remez, Fellowes, \& Rubin, 1997).

Thus, not all sources of variability (i.e., indexical and allophonic) are created equal and may indeed follow distinct courses of temporal processing.

\section{REFERENCES}

Abercrombie, D. (1967). Elements of General Phonetics. Chicago: Aldine.

Allopenna, P. D., Magnuson, J. S., \& Tanenhaus, M. K. (1998). Tracking the time course of spoken word recognition using eye movements: Evidence for continuous mapping models. Journal of Memory and Language, 38, 419-39.

Biederman, I. \& Cooper, E. E. (1992). Size invariance in visual object priming. Journal of Experimental Psychology: Human Perception and Performance, 18, 122-33.

Burton, M. W., Baum, S. R., \& Blumstein, S. E. (1989). Lexical effects on the phonetic categorization of speech: the role of acoustic structure. Journal of Experimental Psychology: Human Perception and Performance, $15,567-75$.

Church, B. A. \& Schacter, D. L. (1994).

Perceptual specificity of auditory priming: Implicit memory for voice intonation and fundamental frequency. Journal of Experimental Psychology: Learning, Memory, and Cognition, 20, 521-33.

Cluff, M. S. \& Luce, P. A. (1990). Similarity neighborhoods of spoken bisyllabic words. Journal of Experimental Psychology: Human Perception and Performance, 16, 551-63.

Connine, C. M., Blasko, D. G., \& Titone, D. (1993). Do the beginnings of words have a special status in auditory word recognition? Journal of Memory and Language, 32, 193-210.

Cooper, L. A., Schacter, D. L., Ballesteros, S., \& Moore, C. (1992). Priming and recognition of transformed three-dimensional objects: Effects of size and reflection. Journal of Experimental Psychology: Learning, Memory, and Cognition, 18, 43-57.

Creelman, C. D. (1957). The case of the unknown talker. Journal of the Acoustical Society of America, 29, 655.

Cutler, A. \& Norris, D. G. (1988). The role of strong syllables in segmentation for lexical access. Journal of Experimental Psychology: Human Perception and Performance, 14, 113-21.

Cutler, A., Norris, D., \& Williams, J. N. (1987). A note on the role of phonological expectation in speech segmentation. Journal of Memory and Language, 26, 480-7. 
Fourcin, A. J. (1968). Speech-source interference. IEEE Trans. Audio Electroacoustics, ACC-16, 65-7.

Frauenfelder, U. \& Peeters, G. (1990). Lexical segmentation in TRACE: An exercise in simulation. In G. T. Altmann (ed.), Cognitive Models of Speech Processing (pp. 50-86). Cambridge, MA: MIT Press.

Gaskell, M. G. \& Marslen-Wilson, W. D. (1997). Integrating form and meaning: A distributed model of speech perception. Language and Cognitioe Processes, 12, 613-56.

Gaskell, M. G. \& Marslen-Wilson, W. D. (1999). Ambiguity, competition, and blending in spoken word recognition. Cognitive Science, 23, 439-62.

Gaskell, M. G. \& Marslen-Wilson, W. D. (2002). Representation and competition in the perception of spoken words. Cognitive Psychology, 45, 220-66.

Goldinger, S. D. (1996). Words and voices: Episodic traces in spoken word identification and recognition memory. Joumal of Experimental Psychology: Learning, Memory, and Cognition, 22, 1166-83.

Goldinger, S. D. (1998). Echoes of echoes? An episodic theory of lexical access. Psychological Revicw, 105(2), 251-79.

Goldinger, S. D., Luce, P. A., \& Pisoni, D. B. (1989). Priming lexical neighbors of spoken words: Effects of competition and inhibition. Journal of Memory and Language, 28, 501-18.

Goldinger, S. D., Luce, P. A., Pisoni, D. B., \& Marcario, J. K. (1992). Form-based priming in spoken word recognition: The roles of competitive activation and response biases. Journal of Experimental Psychology: Learning, Memory, and Cognition, 18, 1210-37.

Goldinger, S. D., Pisoni, D. B., \& Logan, J. S. (1991). On the nature of talker variability effects on recall of spoken word lists. Journal of Experimental Psychology: Learning, Memory, and Cognition, 17(1), 152-62.

Grossberg, S. \& Myers, C. W. (2000). The resonant dynamics of speech perception: Interword integration and duration- dependent backward effects. Psychological Review, 107, 735-67.

Grossberg, S., Boardman, I., \& Cohen, M. (1997). Neural dynamics of variable-rate speech categorization. Journal of Experimental Psychology: Human Perception and Performance, 23, 483-503.

Hintzman, D. L. (1986). "Schema abstraction" in a multiple-trace memory model. Psychological Review, 93, 411-28.

Hintzman, D. L. \& Caulton, D. A. (1997). Recognition memory and modality judgments: A comparison of retrieval dynamics. Joumal of Memory and Language, 37, 1-23.

Hintzman, D. L. \& Curran, T. (1997). Comparing retrieval dynamics in recognition memory and lexical decision. Journal of Experimental Psychology: General, 126, 228.

Jusczyk, P. W. \& Luce, P. A. (2002). Speech perception and spoken word recognition: Past and present. Ear and Hearing, 23, 1-40.

Kirk, K. I., Pisoni, D. B., \& Miyamoto, R. C. (1997). Effects of stimulus variability on speech perception in listeners with hearing impairment. Journal of Speech, Language, and Hearing Research, 40, 1395-405.

Ladefoged, P. (2000). A Course in Phonetics (5th edition). San Diego: Harcourt Brace Jovanovich.

Luce, P. A. \& Lyons, E. A. (1998). Specificity of memory representations for spoken words. Memory $\&$ Cognition, $26,708-15$.

Luce, P. A. \& Pisoni, D. B. (1998). Recognizing spoken words: The neighborhood activation model. Ear and Hearing, 19, 1-36.

Luce, P. A., Goldinger, S. D., Auer, E. T., \& Vitevitch, M. S. (2000). Phonetic priming, neighborhood activation, and PARSYN. Perception $\mathcal{E}$ Psychophysics, 62, 615-25.

Luce, P. A., McLennan, C., \& CharlesLuce, J. (2003). Abstractness and specificity in spoken word recognition: Indexical and allophonic variability in long-term repetition priming. In 
J. Bowers \& C. Marsolek (eds.), Rethinking Implicit Memory (pp. 197-214).

Oxford: Oxford University Press.

Marslen-Wilson, W. D. (1989). Access and integration: Projecting sound onto meaning. In W. D. Marslen-Wilson (ed.), Lexical Access and Representation (pp. 3-24). Cambridge, MA: Bradford.

Marslen-Wilson, W. D. \& Tyler, L. K. (1980). The temporal structure of spoken language understanding. Cognition, 8 , 1-71.

Marslen-Wilson, W. D. \& Warren, P. (1994). Levels of perceptual representation and process in lexical access: Words, phonemes, and features. Psychological Review, 101, 653-75.

Marslen-Wilson, W. D. \& Welsh, A. (1978). Processing interactions and lexical access during word recognition in continuous speech. Cognitive Psychology, 10, 29-63.

Martin, C. S., Mullennix, J. W., Pisoni, D. B., \& Summers, W. V. (1989). Effects of talker variability on recall of spoken word lists. Journal of Experimental Psychology: Learning, Memory, and Cognition, 15(4), 676-84.

McClelland, J. L. \& Elman, J. L. (1986). The TRACE model of speech perception. Cognitive Psychology, 18, 1-86.

McLennan, C., Luce, P. A., \& CharlesLuce, J. (2003) Representation of lexical form. Journal of Experimental Psychology: Learning, Memory, \& Cognition, 29, 52-53.

McQueen, J. M. (1991). The influence of the lexicon on phonetic categorization: Stimulus quality and word-final ambiguity. Journal of Experimental Psychology: Human Perception and Performance, 17, 433-43.

McQueen, J. M., Norris, D., \& Cutler, A. (1994). Competition in spoken word recognition - spotting words in other words. Journal of Experimental Psychology: Learning, Memory, and Cognition, 20, 621-38.

McQueen, J. M., Norris, D., \& Cutler, A. (1999). Lexical influence in phonetic decision making: Evidence from subcategorical mismatches. Journal of
Experimental Psychology: Human

Perception and Performance, 25, 1363-89.

Mullennix, J. W., Pisoni, D. B., \& Martin, C. S. (1989). Some effects of talker variability on spoken word recognition. Journal of the Acoustical Society of America, 85, 365-78.

Norris, D. (1994). Shortlist: A connectionist model of continuous speech recognition. Cognition, 52, 189-234.

Norris, D., McQueen, J., \& Cutler, A. (1995). Competition and segmentation in spoken word recognition. Journal of Experimental Psychology: Learning, Memory, and Cognition, 21, 1209-28.

Norris, D., McQueen, J., \& Cutler, A. (2000). Merging information in speech recognition: Feedback is never necessary. Brain and Behavioral Sciences, 23, 299-325.

Palmeri, T. J., Goldinger, S. D., \& Pisoni, D. B. (1993). Episodic encoding of voice attributes and recognition memory for spoken words. Journal of Experimental Psychology: Learning, Memory, and Cognition, 19(2), 309-28.

Patterson, D. \& Connine, C. M. (2001). Variant frequency in flap production: A corpus analysis of variant frequency in American English flap production. Phonetica, 58, 254-75.

Peters, R. W. (1955). The relative intelligibility of single-voice and multiple-voice messages under various conditions of noise. Joint Project Report, 56 (pp. 1-9). US Naval School of Aviation Medicine, Pensacola, Florida.

Pisoni, D. B. (1990). Effects of talker variability on speech perception: Implications for current research and theory. In H. Fujisaki (ed.), Proceedings of the 1990 International Conference on Spoken Language Processing (pp. 1399-407). Kobe, Japan, November 18-22.

Pisoni, D. B. (1992a). Some comments on invariance, variability, and perceptual normalization in speech perception. Proceedings of the International Conference on Spoken Language Processing (pp. 587-90). Banff, Canada. 
Pisoni, D. B. (1992b). Talker normalization in speech perception. In Y. Tohkura, E. Vatikiotis-Bateson, \& Y. Sagisaka (eds.), Specch Perception, Production, and Linguistic Structure (pp. 143-51). Tokyo, Japan: Ohmsha Press.

Pisoni, D. B. (1997). Some thoughts on "normalizaton" in speech perception. In K. Johnson \& J. W. Mullennix (eds.), Talker Variability in Specel Processing (pp. 9-32). San Diego, CA: Academic Press.

Pisoni, D. B. \& Luce, P'. A. (1987). Acoustic-phonetic representations in word recognition. Cognition, 25, 1-52.

Remez, R. E., Fellowes, J., \& Rubin, P. E. (1997). Talker identification based on phonetic information. Jowrnal of Experimental Psychology: Human Perception and Performance, 23, 651-66.

Ryalls, B. O. \& Pisoni, D. B. (1997). The effect of talker variability on word recognition in preschool children. Developmontal Psychology, 33, 441-52.

Schacter, D. L. \& Church, B. A. (1992). Auditory priming: Implicit and explicit memory for words and voices. Journal of Experimental Psychology: Learning, Memory, and Cognition, 18, 915-30.
Sheffert, S. M. (1998). Voice-specificity effects on auditory word priming. Memory \& Cognition, 26, 591-8.

Streeter, L. A. \& Nigro, G. N. (1979). The role of medial consonant transitions in word perception. Journal of the Acoustical Society of America, 65, 1533-41.

Verbrugge, R. R., Strange, W., Shankweiler, D. P., \& Edman, T. R. (1976). What information enables a listener to map a talker's vowel space? Journal of the Acoustical Society of America, 60, 198-212.

Vitevitch, M. S. \& Luce, P. A. (1998). When words compete: Levels of processing in spoken word perception. Psychological Science, 9, 325-329.

Vitevitch, M. S. \& Luce, P. A. (1999). Probabilistic phonotactics and neighborhood activation in spoken word recognition. Journal of Memory and Language, 40, 374-408.

Whalen, D. H. (1984). Subcategorical phonetic mismatches slow phonetic judgments. Perception \& Psychophysics, $35,49-64$.

Whalen, D. H. (1991). Subcategorical phonetic mismatches and lexical access. Perception \& Psychophysics, 50, 351-60. 